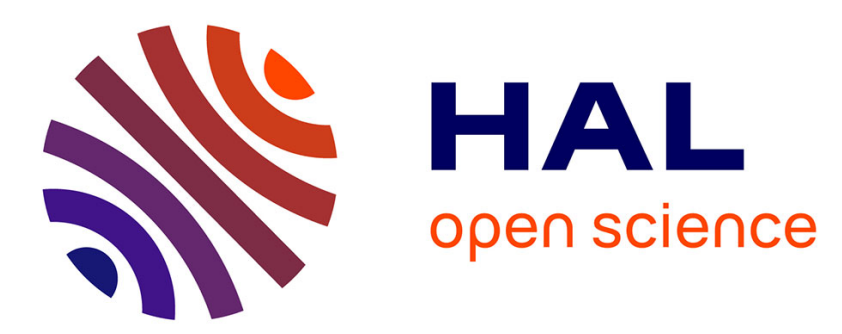

\title{
Improved observer design for heat equation with constant measurement delay via Legendre polynomials
}

\author{
Jin Zhang, Wen Kang, Emilia Fridman, Alexandre Seuret
}

\section{To cite this version:}

Jin Zhang, Wen Kang, Emilia Fridman, Alexandre Seuret. Improved observer design for heat equation with constant measurement delay via Legendre polynomials. 59th IEEE Conference on Decision and Control (CDC 2020), Dec 2020, Jeju (virtual), South Korea. pp.4448-4453, 10.1109/CDC42340.2020.9304502 . hal-03191064

\section{HAL Id: hal-03191064 \\ https://hal.laas.fr/hal-03191064}

Submitted on 6 Apr 2021

HAL is a multi-disciplinary open access archive for the deposit and dissemination of scientific research documents, whether they are published or not. The documents may come from teaching and research institutions in France or abroad, or from public or private research centers.
L'archive ouverte pluridisciplinaire HAL, est destinée au dépôt et à la diffusion de documents scientifiques de niveau recherche, publiés ou non, émanant des établissements d'enseignement et de recherche français ou étrangers, des laboratoires publics ou privés. 


\title{
Improved observer design for heat equation with constant measurement delay via Legendre polynomials
}

\author{
Jin Zhang, Wen Kang, Emilia Fridman and Alexandre Seuret
}

\begin{abstract}
In this paper, we present improved results on observer design for $1 \mathrm{D}$ heat equation. We first introduce an observer under delayed spatially point measurements that leads to an error heat equation with time-delay. Inspired by recent developments in the area of delayed ODEs, we propose novel Lyapunov functionals based on the Legendre polynomials. Then, new Bessel-Legendre (BL) inequalities are provided to derive sufficient stability conditions in the form of linear matrix inequalities (LMIs) that are parameterized by the degree of the polynomials. Finally, a numerical example illustrates the efficiency of the results that allow to enlarge the value of delay preserving the stability by more than $20 \%$.
\end{abstract}

\section{INTRODUCTION}

Estimation of partial differential equations (PDEs) is becoming an active research topic [1]. It is of interest to design observers using delayed measurements. Constructive conditions in the form of LMIs for estimation of PDEs under delayed measurements that are applicable to the performance (e.g. exponential decay rate) analysis have been presented in [2], [3], [4].

To enlarge the delay size, the concept of chain of subobservers was recently extended to heat equation in [5]. However, construction of these observers involves serious computational complexity when solving chain of subobservers in the form of PDEs. The objective of the current note is derivation of less conservative LMI conditions for the stability analysis of the delayed heat equation. Such conditions will allow to reduce the order of chain of subobservers.

In the case of ODEs with time-delay, Jensen inequality [6], [7] and Wirtinger-based integral inequality [8] were usually employed. Several contributions to derive less conservative integral inequalities for time-delay systems were provided in [9], [10]. Recently, a novel integral inequality so-called Bessel-Legendre (BL) inequality that encompasses Jensen inequality and Wirtinger-based integral inequality as particular cases was introduced in [11]. The latter paper presented a hierarchy of LMI conditions that are competitive with [6],

\footnotetext{
This work was supported in part by the Planning and Budgeting Committee (PBC) Fellowship from the Council for Higher Education, Israel, in part by Israel Science Foundation under Grant 1128/14 and Grant 673/19, in part by Joint Research Project HetCPS, Ministry of Science \& Technology of Israel and CNRS, France.

J. Zhang and E. Fridman are with the School of Electrical Engineering, Tel Aviv University, Tel Aviv 69978, Israel (e-mail addresses: zhangjin1116@126.com, emiliadeng.tau.ac.il).

W. Kang is with the School of Automation and Electrical Engineering, University of Science and Technology Beijing, Beijing 100083, China (email address: kangwen@ams. ac.cn).

A. Seuret is with LAAS-CNRS, Université de Toulouse, CNRS, France (e-mail address: aseuret@laas.fr).
}

[7], [8] in terms of conservatism and of complexity. Stability analysis of a coupled ODE-heat PDE was presented in [12] via a new BL inequality.

Following the idea of [11], we provide new BL inequalities by using Legendre polynomials and their properties. Then, we propose novel Lyapunov functionals to derive sufficient stability conditions in the form of LMIs that are parameterized by the degree of the polynomials. Finally, a numerical example illustrates that our LMIs lead to an improvement over $20 \%$. This implies that our results allow to reduce the order of chain of sub-observers considered in [5] by more than $20 \%$.

Notation. Throughout the paper, the subindexes denote the corresponding partial derivatives, $\operatorname{He}(X)$ denotes the expression $X+X^{T}, L^{2}(0, l)$ stands for the Hilbert space of square integrable scalar functions $z(x)$ on $(0, l)$ with the norm $\|z(x)\|_{L^{2}(0, l)}=\sqrt{\int_{0}^{l} z^{2}(x) d x}, H^{1}(0, l)$ is the Sobolev space of absolutely continuous scalar functions $z:[0, l] \rightarrow R$ with $\frac{d z}{d \xi} \in L^{2}(0, l)$, and $H^{2}(0, l)$ is the Sobolev space of scalar functions $z:[0, l] \rightarrow R$ with absolutely continuous $\frac{d z}{d \xi}$ and with $\frac{d^{2} z}{d \xi^{2}} \in L^{2}(0, l)$.

We now present a useful inequality:

Lemma 1: (Wirtinger Inequality [13]) For given scalars $a<b$, consider a scalar function $g \in \mathscr{H}^{1}(a, b)$ such that $g(a)=g(b)=0$. Then the following inequality holds:

$$
\int_{a}^{b} g^{2}(x) d x \leq \frac{(b-a)^{2}}{\pi^{2}} \int_{a}^{b}\left[\frac{d g(x)}{d x}\right]^{2} d x .
$$

\section{PROBLEM FORMULATION}

\section{A. System description}

Consider a semilinear reaction-diffusion equation

$$
\begin{gathered}
z_{t}(x, t)=\frac{\partial}{\partial x}\left[a(x) z_{x}(x, t)\right]+f(z(x, t), x, t), \\
t \geq 0, \quad x \in[0, l], \quad l>0
\end{gathered}
$$

under the Dirchlet boundary conditions

$$
z(0, t)=z(l, t)=0,
$$

where $z(x, t) \in \mathbb{R}$ is the state with initial condition $z(x, 0)=$ $z_{0}(x), a$ and $f$ are functions of class $C^{1}$. These functions satisfy the inequalities $a>a_{0}>0$ and

$$
\phi_{m} \leq f_{z}(z, x, t) \leq \phi_{M}, \quad \forall(z, x, t) \in \mathbb{R} \times[0, l] \times[0, \infty),
$$

where $a_{0}, \phi_{m}$ and $\phi_{M}$ are known bounds.

As in [2], we assume that the points $0=x_{0}<x_{1}<\cdots<$ $x_{N}=l$ divide $[0, l]$ into $N$ sub-intervals, whose widths of each 
interval are not necessarily equal but are upper bounded by some constant $\Delta>0$ :

$$
0<x_{j+1}-x_{j}=\Delta_{j} \leq \Delta
$$

It is assumed that $N$ sensors are placed in the middle of each interval $\left[x_{j}, x_{j+1}\right)$ :

$$
\bar{x}_{j}=\frac{x_{j+1}+x_{j}}{2}, \quad j=0, \ldots, N-1,
$$

and that the measurement is delayed by a constant delay $h>0$. Then, delayed spatially point measurements of the state are provided by $N$ sensors distributed over the whole domain $[0, l]$ :

$$
y_{j}(t)=\left\{\begin{array}{ll}
0, & \text { if } t<h, \\
z\left(\bar{x}_{j}, t-h\right), & \text { if } t \geq h,
\end{array} \quad j=0, \ldots, N-1\right.
$$

Our objective is to construct an observer for (2) under the Dirichlet boundary conditions (3) by employing delayed spatially point measurements (7), and to formulate improve stability conditions for the exponential convergence of the estimation error in terms of LMIs.

\section{B. Observer design}

We suggest a nonlinear observer of the form

$$
\begin{aligned}
\hat{z}_{t}(x, t)= & \frac{\partial}{\partial x}\left[a(x) \hat{z}_{x}(x, t)\right]+f(\hat{z}(x, t), x, t) \\
& +K\left(y_{j}(t)-\hat{z}\left(\bar{x}_{j}, t-h\right)\right), \\
t \geq 0, \quad & x_{j} \leq x<x_{j+1}, \quad j=0, \ldots, N-1
\end{aligned}
$$

under the Dirchlet boundary conditions

$$
\hat{z}(0, t)=\hat{z}(l, t)=0
$$

where $\hat{z}(x, t)=0, t \in[-h, 0]$ and $K>0$ is the scalar observer gain.

For the estimation error $e(x, t)=z(x, t)-\hat{z}(x, t)$, via (2) and (8) we obtain the PDE

$$
\begin{aligned}
& e_{t}(x, t)= \frac{\partial}{\partial x}\left[a(x) e_{x}(x, t)\right]+\phi \cdot e(x, t)-K e(x, t-h) \\
&+K \int_{\bar{x}_{j}}^{x} e \xi(\xi, t-h) d \xi, \\
& t \geq 0, \quad x_{j} \leq x<x_{j+1}, \quad j=0, \ldots, N-1
\end{aligned}
$$

under the Dirichlet boundary conditions

$$
e(0, t)=e(l, t)=0,
$$

and with initial condition $e(x, t)=z_{0}(x), t \in[-h, 0]$.

Here $\phi \cdot e(x, t)=f(z(x, t), x, t)-f(z(x, t)-e(x, t), x, t)$ with

$$
\phi=\phi(z, e, x, t)=\int_{0}^{1} \frac{\partial}{\partial z} f(z+(\theta-1) e, x, t) d \theta .
$$

From (4), it follows that

$$
\phi_{m} \leq \phi \leq \phi_{M}, \quad \forall(z, e, x, t) \in \mathbb{R} \times \mathbb{R} \times[0, l] \times[0, \infty)
$$

\section{Well-posedness of (10)}

Let $H=L^{2}(0, l)$ be the Hilbert space with the norm $\|\cdot\|_{L^{2}(0, l)}$ and with the scalar product $\langle\cdot, \cdot\rangle$. We define an unbounded linear operator $A: \mathscr{D}(A) \subset H \rightarrow H$ as follows:

$$
\left\{\begin{array}{l}
A=\frac{\partial\left[a(x) \frac{\partial}{\partial x}\right]}{\partial x}, \\
\mathscr{D}(A)=H^{2}(0, l) \cap H_{0}^{1}(0, l),
\end{array}\right.
$$

where $H_{0}^{1}(0, l)=\left\{z \in H^{1}(0, l): z(0)=z(l)=0\right\}$. It is wellknown that $A$ is a dissipative operator, and $A$ generates an exponentially stable semigroup. The domain $H_{1}=\mathscr{D}(A)=$ $A^{-1} H$ forms another Hilbert space with the graph inner product $\langle x, y\rangle_{1}=\langle A x, A y\rangle, \forall x, y \in H_{1}$. The domain $\mathscr{D}(A)$ is dense in $H$. Operator $-A$ is positive implying that its square root $(-A)^{\frac{1}{2}}$ with $H_{\frac{1}{2}}=\mathscr{D}\left((-A)^{\frac{1}{2}}\right)=H_{0}^{1}(0, l)$ is well defined. The norm of $H_{\frac{1}{2}}$ is endowed by the induced inner product:

$$
\begin{aligned}
& \langle u, v\rangle_{\frac{1}{2}}=\left\langle(-A)^{\frac{1}{2}} u,(-A)^{\frac{1}{2}} v\right\rangle, \quad \forall u, v \in H_{\frac{1}{2}}, \\
& \|f\|_{H_{\frac{1}{2}}}=\left[\int_{0}^{l}\left|f^{\prime}(x)\right|^{2} d x\right]^{\frac{1}{2}}, \quad \forall f \in H_{\frac{1}{2}} .
\end{aligned}
$$

Note that $H_{\frac{1}{2}}$ norm is equivalent to the inherent norm $\|\cdot\|_{H_{1}}$ of Sobolev space $H^{1}(0, l)$. Then, we have $H_{1} \subset H_{\frac{1}{2}} \subset H$, densely and with continuous embedding. All relevant materials on fractional operator degrees can be found in [14] (see pp. 81-83).

We will use the step method for solution of time-delay systems [15], [16]. While being viewed over the time segment $[0, h]$, system (10) can be rewritten as the differential equation given by

$$
\left\{\begin{array}{l}
\frac{d}{d t} e(\cdot, t)=A e(\cdot, t)+F(e(\cdot, t)), \\
e(x, \theta)=z_{0}(x), \quad \theta \in[-h, 0]
\end{array}\right.
$$

subject to

$$
F(e(\cdot, t))=\phi \cdot e(x, t)+K z_{0}\left(\bar{x}_{j}\right), \quad x \in\left[x_{j}, x_{j+1}\right) .
$$

Since $\phi \in C^{1}$ and $\phi$ is bounded, it follows from (16) that $F$ is nonlinear but is globally Lipschitz continuous. Thus, by Theorem 3.3.3 of [17], we obtain that for any $z_{0} \in H_{0}^{1}(0, l)$, (15) has a unique strong solution $e(\cdot, t) \in H_{0}^{1}(0, l)$ on the interval $[0, h]$.

The same line of reasoning is applied step-by-step to the time segments $[h, 2 h],[2 h, 3 h], \ldots$ (see, e.g. [16]). Following this procedure, we find that $e(\cdot, t)$ is continuous differentiable on the point $h, 2 h, \ldots$ Therefore, there exists a unique strong solution $e(\cdot, t)$ of (10) for all $t \geq 0$ with the initial condition $e(x, \theta)=z_{0}(x) \in H_{0}^{1}(0, l), \theta \in[-h, 0]$.

\section{MAIN RESULTS}

In this section, we will derive improved stability conditions for the delayed heat equation (10) via new BL inequalities. First, let us recall the definition of Legendre polynomials. 


\section{A. Legendre polynomials}

As in [11], we consider here shifted Legendre polynomials over interval $[-h, 0]$

$$
\mathscr{L}_{k}(s)=(-1)^{k} \sum_{i=0}^{k} p_{i}^{k}\left(\frac{s+h}{h}\right)^{i}, \quad \forall k \in \mathbb{N}_{0}
$$

with $p_{i}^{k}=(-1)^{i}\left(\begin{array}{c}k \\ i\end{array}\right)\left(\begin{array}{c}k+i \\ i\end{array}\right)$. The notation $\left(\begin{array}{l}k \\ i\end{array}\right)$ refers to the binomial coefficients given by $\frac{k !}{(k-i) ! i !}$. Note that the Legendre polynomials described by (17) form an orthogonal sequence since the inner product satisfies

$$
\int_{-h}^{0} \mathscr{L}_{k}(s) \mathscr{L}_{i}(s) d s=\frac{h}{2 k+1} \delta_{k i}, \quad \forall k, i \in \mathbb{N}_{0},
$$

where $\delta_{k i}$ is the Kronecker delta (that equals to 1 if $k=i$ and to 0 otherwise). The boundary values are as follows:

$$
\mathscr{L}_{k}(0)=1, \quad \mathscr{L}_{k}(-h)=(-1)^{k}, \quad \forall k \in \mathbb{N}_{0} .
$$

Moreover, the following relation holds:

$$
\frac{d}{d s} \mathscr{L}_{k}(s)= \begin{cases}0, & \text { if } k=0, \\ \sum_{i=0}^{k-1} \frac{2 i+1}{h}\left(1-(-1)^{k+i}\right) \mathscr{L}_{i}(s), & \text { if } k \geq 1 .\end{cases}
$$

Let us introduce the projection components of the error heat equation (10) onto Legendre polynomials. These components are given by

$$
\Omega_{k}(x, t)=\int_{-h}^{0} \mathscr{L}_{k}(s) e(x, t+s) d s, \quad k \in \mathbb{N}_{0} .
$$

Differentiating $\Omega_{k}(x, t)$ with respect to $t$ leads to

$$
\frac{\partial}{\partial t} \Omega_{k}(x, t)=\int_{-h}^{0} \mathscr{L}_{k}(s) \frac{\partial}{\partial t} e(x, t+s) d s, \quad k \in \mathbb{N}_{0} .
$$

By using (19) and (20), and integrating by parts, we have

$$
\begin{aligned}
\frac{\partial}{\partial t} \Omega_{k}(x, t)= & \left.\mathscr{L}_{k}(s) e(x, t+s)\right|_{s=-h} ^{0} \\
& -\int_{-h}^{0}\left[\frac{d}{d s} \mathscr{L}_{k}(s)\right] e(x, t+s) d s \\
= & e(x, t)+(-1)^{k+1} e(x, t-h) \\
& -\int_{-h}^{0}\left[\frac{d}{d s} \mathscr{L}_{k}(s)\right] e(x, t+s) d s \\
= & \Gamma_{n}(k) \eta_{n}(x, t), \quad k=0, \ldots, n, \quad n \in \mathbb{N}_{0},
\end{aligned}
$$

where

$$
\begin{aligned}
& \Gamma_{n}(k)= \begin{cases}{\left[1,0,(-1)^{k+1}, 0\right],} & \text { if } n=0, \\
{\left[1,0,(-1)^{k+1}, 0, \gamma_{n k}^{0}, \ldots, \gamma_{n k}^{n-1}\right],} & \text { if } n \geq 1,\end{cases} \\
& \gamma_{n k}^{i}= \begin{cases}-\frac{2 i+1}{h}\left(1-(-1)^{k+i}\right), & \text { if } i \leq k, \\
0, & \text { if } i>k,\end{cases}
\end{aligned}
$$

and

$$
\eta_{n}(x, t)=\left\{\begin{array}{r}
{\left[e(x, t), e_{t}(x, t), e(x, t-h), \int_{\bar{x}_{j}}^{x} e_{\xi}(\xi, t-h) d \xi\right]^{T},} \\
\text { if } n=0, \\
{\left[e(x, t), e_{t}(x, t), e(x, t-h), \int_{\bar{x}_{j}}^{x} e_{\xi}(\xi, t-h) d \xi\right.} \\
\left.\Omega_{0}(x, t), \cdots, \Omega_{n-1}(x, t)\right]^{T}, \quad \text { if } n \geq 1 .
\end{array}\right.
$$

The relation given by (23) is very useful to derive BL inequality (see e.g. the proof of Lemma 3 ) and also for the later stability analysis.

\section{B. Bessel-Legendre inequalities}

Based on the Legendre polynomials and an application of Bessel's inequality [18], we obtain the following lemma:

Lemma 2: Let $e \in L^{2}\left([-h, 0] ; L^{2}(0, l)\right), h>0$ and $l>0$. The integral inequality

$$
\int_{0}^{l} \int_{-h}^{0} e^{2}(x, t+s) d s d x \geq \frac{1}{h} \sum_{k=0}^{n}(2 k+1) \int_{0}^{l} \Omega_{k}^{2}(x, t) d x
$$

holds for all $n \in \mathbb{N}_{0}$, where $\Omega_{k}(x, t), k=0, \ldots, n$ are given by (21).

Proof: Consider a function $e \in L^{2}\left([-h, 0] ; L^{2}(0, l)\right)$, and define the error vector $\varepsilon_{n}(x, t+s), n \in \mathbb{N}_{0}$ given by

$$
\varepsilon_{n}(x, t+s)=e(x, t+s)-\frac{1}{h} \sum_{k=0}^{n}(2 k+1) \mathscr{L}_{k}(s) \Omega_{k}(x, t) .
$$

From its definition, $\varepsilon_{n}(x, t+s)$ is in $L^{2}\left([-h, 0] ; L^{2}(0, l)\right)$ and the integral $\int_{0}^{l} \int_{-h}^{0} \varepsilon_{n}^{2}(x, t+s) d s d x$ exists. From the orthogonal property of the Legendre polynomials, via (21) we easily get

$$
\begin{aligned}
& \int_{0}^{l} \int_{-h}^{0} \varepsilon_{n}^{2}(x, t+s) d s d x=\int_{0}^{l}\left\{\int_{-h}^{0} e^{2}(x, t+s) d s\right. \\
& \quad-\frac{2}{h} \sum_{k=0}^{n}(2 k+1)\left[\int_{-h}^{0} \mathscr{L}_{k}(s) e(x, t+s) d s\right] \Omega_{k}(x, t) \\
& \left.\quad+\frac{1}{h^{2}} \sum_{k=0}^{n}(2 k+1)^{2} \int_{-h}^{0} \mathscr{L}_{k}^{2}(s) \Omega_{k}^{2}(x, t) d s\right\} d x \\
& =\int_{0}^{l}\left\{\int_{-h}^{0} e^{2}(x, t+s) d s-\frac{1}{h} \sum_{k=0}^{n}(2 k+1) \Omega_{k}^{2}(x, t)\right\} d x .
\end{aligned}
$$

Noting that the left hand side of the latter equation is positive definite, we finally arrive at (26), which concludes the proof.

For the delay-dependent analysis of delayed heat equation (10), we are interested to derive a lower bound of $\int_{0}^{l} \int_{-h}^{0} e_{s}^{2}(x, t+s) d s d x$. The next lemma addresses this particular problem:

Lemma 3: Let $e_{s} \in L^{2}\left([-h, 0] ; L^{2}(0, l)\right), h>0$ and $l>0$. The integral inequality

$$
\begin{aligned}
& \int_{0}^{l} \int_{-h}^{0} e_{s}^{2}(x, t+s) d s d x \\
& \geq \frac{1}{h} \sum_{k=0}^{n}(2 k+1) \int_{0}^{l}\left[\Gamma_{n}(k) \eta_{n}(x, t)\right]^{2} d x
\end{aligned}
$$

holds for all $n \in \mathbb{N}_{0}$, where $\Gamma_{n}(k), k=0, \ldots, n$ and $\eta_{n}(x, t)$ are given by (24) and (25).

Proof: By using Lemma 2, we obtain

$$
\int_{0}^{l} \int_{-h}^{0} e_{s}^{2}(x, t+s) d s d x \geq \frac{1}{h} \sum_{k=0}^{n}(2 k+1) \int_{0}^{l}\left[\frac{\partial}{\partial t} \Omega_{k}(x, t)\right]^{2} d x .
$$

Replacing $\frac{\partial}{\partial t} \Omega_{k}(x, t)$ by its expression (23) using the vectors $\Gamma_{n}(k), k=0, \ldots, n$ and $\eta_{n}(x, t)$ yields (29). This completes the proof. 


\section{Stability analysis}

Guided by these new BL inequalities, we consider an augmented Lyapunov functional as follows

$$
V_{n}(t)=V_{P_{n}}(t)+V_{p_{2}}(t)+V_{s_{1}}(t)+V_{s_{2}}(t)+V_{r}(t), \quad n \in \mathbb{N}_{0},
$$

where

$$
\begin{aligned}
& V_{P_{n}}(t)=\int_{0}^{l} \zeta_{n}^{T}(x, t) P_{n} \zeta_{n}(x, t) d x, \\
& V_{p_{2}}(t)=p_{2} \int_{0}^{l} a(x) e_{x}^{2}(x, t) d x, \\
& V_{s_{1}}(t)=s_{1} \int_{0}^{l} \int_{t-h}^{t} e^{2 \alpha(s-t)} e^{2}(x, s) d s d x, \\
& V_{s_{2}}(t)=s_{2} \int_{0}^{l} \int_{t-h}^{t} e^{2 \alpha(s-t)} e_{x}^{2}(x, s) d s d x, \\
& V_{r}(t)=h r \int_{0}^{l} \int_{t-h}^{t} e^{2 \alpha(s-t)}(s-t+h) e_{s}^{2}(x, s) d s d x
\end{aligned}
$$

with

$$
\zeta_{n}(x, t)= \begin{cases}e(x, t), & \text { if } n=0 \\ {\left[e(x, t), \Omega_{0}(x, t), \cdots, \Omega_{n-1}(x, t)\right]^{T},} & \text { if } n \geq 1\end{cases}
$$

and matrix $P_{n} \in \mathbb{R}^{(n+1) \times(n+1)}$, scalars $p_{2}>0, s_{1}>0, s_{2}>$ $0, r>0$ and decay rate $\alpha>0$. The terms $V_{s_{1}}$ and $V_{r}$ are the extensions of the standard Lyapunov functionals [15] for delay-dependent analysis, whereas the term $V_{s_{2}}$ is introduced to compensate $\int_{0}^{l} e_{x}(x, t-h) d x$. For $n=0, V_{n}$ coincides with the Lyapunov functional introduced in [2].

We now present the following stability results with an arbitrary $n \in \mathbb{N}_{0}$ :

Theorem 1: Given scalars $\phi_{M} \geq \phi_{m}, a_{0}>0, h>0, l>0$, $\Delta>0$ and $\alpha>0$, let there exist $P_{n} \in \mathbb{R}^{(n+1) \times(n+1)}$ and scalars $p_{1}>0, p_{2}>0, s_{1}>0, s_{2}>0, r>0$ and $\mu>0$ satisfying the following LMIs

$$
s_{2}<2 a_{0}\left(p_{1}-\alpha p_{2}\right), \quad \mu<e^{-2 \alpha h} s_{2},
$$

and

$$
\Phi_{n}\left(\phi_{m}\right)+U_{n} \leq 0, \quad \Phi_{n}\left(\phi_{M}\right)+U_{n} \leq 0, \quad \Theta_{n}>0,
$$

where

$$
\begin{aligned}
& \Phi_{n}(\phi)= \operatorname{He}\left(G_{n}^{T} P_{n} H_{n}+D_{n}^{T} R_{n}(\phi)\right)+2 \alpha G_{n}^{T} P_{n} G_{n} \\
&+W_{n}-e^{-2 \alpha h} r \sum_{k=0}^{n}(2 k+1) \Gamma_{n}^{T}(k) \Gamma_{n}(k), \\
& U_{n}=\operatorname{diag}\left\{-\frac{\left(2 a_{0}\left(p_{1}-\alpha p_{2}\right)-s_{2}\right) \pi^{2}}{l^{2}}, 0,\right.\left.-\frac{\left(e^{-2 \alpha h} s_{2}-\mu\right) \pi^{2}}{l^{2}}, 0_{1 \times n}\right\}, \\
& \Theta_{n}= \begin{cases}P_{n}, & \text { if } n=0, \\
P_{n}+\frac{1}{h} e^{-2 \alpha h_{1}} s_{1} \operatorname{diag}\{0,1, \ldots, 2 n-1\}, & \text { if } n \geq 1\end{cases}
\end{aligned}
$$

with

$$
\begin{aligned}
& G_{n}=\left[\begin{array}{ccc}
1 & 0_{1 \times 3} & 0_{1 \times n} \\
0_{n \times 1} & 0_{n \times 3} & I_{n}
\end{array}\right], \\
& H_{n}=\left[\begin{array}{llll}
F_{n}^{T} & \Gamma_{n}^{T}(0) & \ldots & \Gamma_{n}^{T}(n-1)
\end{array}\right]^{T}, \\
& F_{n}=\left[\begin{array}{lll}
0 & 1 & 0_{1 \times(n+2)}
\end{array}\right] \text {, } \\
& D_{n}=\left[\begin{array}{lll}
p_{1} & p_{2} & 0_{1 \times(n+2)}
\end{array}\right] \text {, } \\
& R_{n}(\phi)=\left[\begin{array}{lllll}
\phi & -1 & -K & K & 0_{1, n}
\end{array}\right] \text {, } \\
& W_{n}=\operatorname{diag}\left\{s_{1}, h^{2} r,-e^{-2 \alpha h} s_{1},-\mu \frac{\pi^{2}}{\Delta^{2}}, 0_{1 \times n}\right\},
\end{aligned}
$$

and $\Gamma_{n}(k)(k=0, \ldots, n)$ given by (24). Then the delayed heat equation (10) under the Dirichlet boundary conditions (11) is exponentially stable with a decay rate $\alpha>0$.

Proof: Choose the Lyapunov function $V_{n}(t)$ given by (31). Using Lemma 2 gives a lower bound of the term $V_{s_{1}}$ as follows

$$
\begin{aligned}
V_{s_{1}}(t) & \geq e^{-2 \alpha h} s_{1} \int_{0}^{l} \int_{-h}^{0} e^{2}(x, t+s) d s d x \\
& \geq \frac{s_{1}}{h} e^{-2 \alpha h} \sum_{k=0}^{n-1}(2 k+1) \int_{0}^{l} \Omega_{k}^{2}(x, t) d x .
\end{aligned}
$$

Thus, the positive definiteness of $V_{n}$ results from the condition $\Theta_{n}>0$ given by (33).

Differentiating $V_{P_{n}}(t)$ along (10), via (23) we obtain

$$
\begin{aligned}
\dot{V}_{P_{n}}(t)+2 \alpha V_{P_{n}}(t)= & 2 \int_{0}^{l} \zeta_{n}^{T}(x, t) P_{n} \dot{\zeta}_{n}(x, t) d x \\
& +2 \alpha \int_{0}^{l} \zeta_{n}^{T}(x, t) P_{n} \zeta_{n}(x, t) d x,
\end{aligned}
$$

where

$$
\dot{\zeta}_{n}(x, t)=\left[\begin{array}{c}
\dot{e}(x, t) \\
\dot{\Omega}_{0}(x, t) \\
\vdots \\
\dot{\Omega}_{n-1}(x, t)
\end{array}\right]=\left[\begin{array}{c}
F_{n} \eta_{n}(x, t) \\
\Gamma_{n}(0) \eta_{n}(x, t) \\
\vdots \\
\Gamma_{n}(n-1) \eta_{n}(x, t)
\end{array}\right]=H_{n} \eta_{n}(x, t) .
$$

For the term $V_{p_{2}}$, We have

$$
\begin{aligned}
\dot{V}_{p_{2}}(t)+2 \alpha V_{p_{2}}(t)= & 2 p_{2} \int_{0}^{l} a(x) e_{x}(x, t) e_{x t}(x, t) d x \\
& +2 \alpha p_{2} \int_{0}^{l} a(x) e_{x}^{2}(x, t) d x .
\end{aligned}
$$

For the first term of the right hand side of (37), we employ the descriptor method [15], where the right-hand side of the following expression is added to $\dot{V}_{n}(t)+2 \alpha V_{n}(t)$ :

$$
\begin{aligned}
0= & 2 \int_{0}^{l}\left[p_{1} e(x, t)+p_{2} e_{t}(x, t)\right]\left\{\frac{\partial}{\partial x}\left[a(x) e_{x}(x, t)\right]\right. \\
& \left.+\phi e(x, t)-K e(x, t-h)-e_{t}(x, t)\right\} d x \\
& +2 K \sum_{j=0}^{N-1} \int_{x_{j}}^{x_{j+1}}\left[p_{1} e(x, t)+p_{2} e_{t}(x, t)\right] \\
& \times \int_{\bar{x}_{j}}^{x} e_{\xi}(\xi, t-h) d \xi d x
\end{aligned}
$$

with some scalar $p_{1}>0$. This avoids substitution of $e_{t}(x, t)$ from (10) into the right-hand side of (36). 
Integration by parts and substitution of the Dirichlet boundary conditions (11) lead to

$$
\begin{aligned}
& 2 \int_{0}^{l}\left[p_{1} e(x, t)+p_{2} e_{t}(x, t)\right] \frac{\partial}{\partial x}\left[a(x) e_{x}(x, t)\right] d x \\
= & \left.2 a(x)\left[p_{1} e(x, t)+p_{2} e_{t}(x, t)\right] e_{x}(x, t)\right|_{x=0} ^{l} \\
& -2 \int_{0}^{l} a(x)\left[p_{1} e_{x}(x, t)+p_{2} e_{x t}(x, t)\right] e_{x}(x, t) d x \\
= & -2 p_{1} \int_{0}^{l} a(x) e_{x}^{2}(x, t) d x-2 p_{2} \int_{0}^{l} a(x) e_{x}(x, t) e_{x t}(x, t) d x .
\end{aligned}
$$

To "compensate" the cross terms in (38) with $\int_{\bar{x}_{j}}^{x} e_{\xi}(\xi, t-$ h) $d \xi$, we here apply S-procedure. Namely, application of Wirtinger's inequality (1) yields

$$
\begin{aligned}
& \int_{x_{j}}^{x_{j+1}}\left[\int_{\bar{x}_{j}}^{x} e_{\xi}(\xi, t-h) d \xi\right]^{2} d x \\
& =\int_{\bar{x}_{j}}^{x_{j+1}}\left[\int_{\bar{x}_{j}}^{x} e_{\xi}(\xi, t-h) d \xi\right]^{2} d x \\
& \quad+\int_{x_{j}}^{\bar{x}_{j}}\left[\int_{\bar{x}_{j}}^{x} e_{\xi}(\xi, t-h) d \xi\right]^{2} d x \\
& \leq \frac{\Delta^{2}}{\pi^{2}} \int_{x_{j}}^{x_{j+1}} e_{x}^{2}(x, t-h) d x, \quad j=0, \ldots, N-1 .
\end{aligned}
$$

Then the following inequality

$$
\begin{aligned}
& \mu \int_{0}^{l} e_{x}^{2}(x, t-h) d x \\
& -\mu \sum_{j=0}^{N-1} \int_{x_{j}}^{x_{j+1}} \frac{\pi^{2}}{\Delta^{2}}\left[\int_{\bar{x}_{j}}^{x} e_{\xi}(\xi, t-h) d \xi\right]^{2} d x \geq 0
\end{aligned}
$$

holds for some constant $\mu>0$.

Moreover, we have

$$
\begin{aligned}
\dot{V}_{s_{1}}(t)+2 \alpha V_{s_{1}}(t)= & s_{1} \int_{0}^{l} e^{2}(x, t) d x \\
& -e^{-2 \alpha h} s_{1} \int_{0}^{l} e^{2}(x, t-h) d x,
\end{aligned}
$$

and

$$
\begin{aligned}
\dot{V}_{s_{2}}(t)+2 \alpha V_{s_{2}}(t)= & s_{2} \int_{0}^{l} e_{x}^{2}(x, t) d x \\
& -e^{-2 \alpha h} s_{2} \int_{0}^{l} e_{x}^{2}(x, t-h) d x .
\end{aligned}
$$

Further by using Lemma 3, we obtain

$$
\begin{aligned}
& \dot{V}_{r}(t)+2 \alpha V_{r}(t)=h^{2} r \int_{0}^{l} e_{t}^{2}(x, t) d x \\
& \quad-h r \int_{0}^{l} \int_{t-h}^{t} e^{2 \alpha(s-t)} e_{s}^{2}(x, s) d s d x \\
& \leq h^{2} r \int_{0}^{l} e_{t}^{2}(x, t) d x \\
& -e^{-2 \alpha h} r \sum_{k=0}^{n}(2 k+1) \int_{0}^{l}\left[\Gamma_{n}(k) \eta_{n}(x, t)\right]^{2} d x .
\end{aligned}
$$

Combining (36), (37), (41)-(43) together, adding the right hand side of (38) to $\dot{V}_{n}(t)+2 \alpha V_{n}(t)$, and applying S- procedure with (40), we have

$$
\begin{aligned}
\dot{V}_{n}(t)+2 \alpha V_{n}(t) \leq & \sum_{j=0}^{N-1} \int_{x_{j}}^{x_{j+1}} \eta_{n}^{T}(x, t) \Phi_{n}(\phi) \eta_{n}(x, t) d x \\
& +s_{2} \int_{0}^{l} e_{x}^{2}(x, t) d x \\
& -2\left(p_{1}-\alpha p_{2}\right) \int_{0}^{l} a(x) e_{x}^{2}(x, t) d x \\
& -\left(e^{-2 \alpha h} s_{2}-\mu\right) \int_{0}^{l} e_{x}^{2}(x, t-h) d x
\end{aligned}
$$

where $\Phi_{n}(\phi)$ is given by (34).

Note that the first inequality of (32) implies $p_{1}-\alpha p_{2}>0$ since $s_{2}>0$ and $a_{0}>0$. Taking into account $a>a_{0}$, we further arrive at

$$
\begin{aligned}
\dot{V}_{n}(t)+2 \alpha V_{n}(t) \leq \sum_{j=0}^{N-1} \int_{x_{j}}^{x_{j+1}} \eta_{n}^{T}(x, t) \Phi_{n}(\phi) \eta_{n}(x, t) d x \\
-\left[2 a_{0}\left(p_{1}-\alpha p_{2}\right)-s_{2}\right] \int_{0}^{l} e_{x}^{2}(x, t) d x \\
\quad-\left(e^{-2 \alpha h} s_{2}-\mu\right) \int_{0}^{l} e_{x}^{2}(x, t-h) d x \\
\leq \sum_{j=0}^{N-1} \int_{x_{j}}^{x_{j+1}} \eta_{n}^{T}(x, t)\left(\Phi_{n}(\phi)+U_{n}\right) \eta_{n}(x, t) d x .
\end{aligned}
$$

Here $U_{n}$ is given by (34). The latter follows from Wirtinger's inequality (1).

Since $\Phi_{n}(\phi)$ given by (34) is affine in $\phi \in\left[\phi_{m}, \phi_{M}\right]$, the feasibility of (33) implies the feasibility of $\Phi_{n}(\phi)+U_{n}<$ 0 for all $\phi \in\left[\phi_{m}, \phi_{M}\right]$. Thus, we have $\dot{V}_{n}(t)+2 \alpha V_{n}(t) \leq 0$ implying the exponential convergence of $V_{n}(t)$ with a decay rate $\alpha>0$.

Remark 1: By following arguments of [11], [19], we can easily show that the derived stability conditions can form a hierarchy of LMI conditions. This will be illustrated via the later example.

\section{NUMERICAL SIMULATIONS}

Consider the following 1D heat equation:

$$
\begin{gathered}
z_{t}(x, t)=z_{x x}(x, t)+1.02 \pi^{2} z(x, t), \\
t \geq 0, \quad x \in[0,1]
\end{gathered}
$$

under the Dirichlet boundary conditions (3). Choose $z(x, 0)=$ $\sin (\pi x)$ and $x_{j+1}-x_{j} \equiv \Delta$ with $\Delta=0.02$ implying the number of sub-intervals $N=50$. The heat equation above is unstable (see Fig. 1).

We suggest the following observer:

$$
\begin{aligned}
& \hat{z}_{t}(x, t)= \hat{z}_{x x}(x, t)+1.02 \pi^{2} \hat{z}(x, t) \\
&+\left(z\left(\bar{x}_{j}, t-h\right)-\hat{z}\left(\bar{x}_{j}, t-h\right)\right), \\
& t \geq 0, \quad x_{j} \leq x<x_{j+1}, \quad j=0, \ldots, 49
\end{aligned}
$$

under the Dirichlet boundary conditions (9). Here $\bar{x}_{j}(j=$ $0, \ldots, 49)$ are given by (6) and $h>0$ is a constant delay.

LMIs of Theorem 1 with various values of $n$ and $\alpha=$ 0 lead to the maximal allowable delay $h_{\max }$ preserving the stability shown in Table I. As expected, better results are obtained as the degree of the polynomial $n$ increases, but at the price of additional decision variables. Moreover, the 


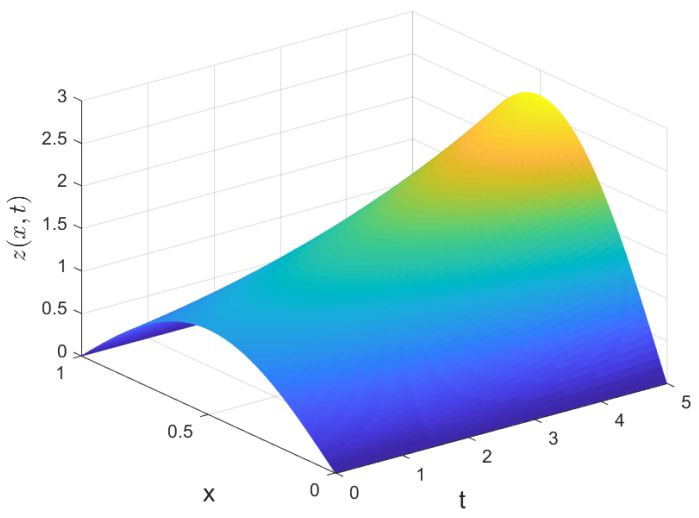

Fig. 1. The state $z(x, t)$.

TABLE I

MAXimal ALLOWABLE DELAY $h_{\max }$ VIA THEOREM 1.

\begin{tabular}{lll}
\hline Theorem 1 & $h_{\max }$ & Number of variables \\
\hline$n=0$ & 0.5215 & 7 \\
$n=1$ & 0.6226 & 9 \\
$n=2$ & 0.6349 & 12 \\
$n=3$ & 0.6389 & 16 \\
$n=4$ & 0.6421 & 21 \\
$n=5$ & 0.6440 & 27 \\
$n=6$ & 0.6449 & 34 \\
$n=7$ & 0.6450 & 42 \\
$n \geq 8$ & 0.6451 & $0.5 n^{2}+1.5 n+7$ \\
\hline
\end{tabular}

maximal allowable delay $h_{\max }$ remains as 0.6451 when $n \geq 8$. Clearly, an improvement over $20 \%$ is achieved by using our new BL inequalities. This implies that our result allows to reduce the order of chain of sub-observers considered in [5] by more than $20 \%$.

The numerical simulations show that the error heat equation under the Dirichlet boundary conditions (11) and with constant delay $h=0.6451$ is stable (see Fig. 2).

\section{CONCLUSiOns}

In this paper, an observer was introduced for 1D heat equation under delayed spatially point measurements. The stability analysis of the resulting error heat equation with time-delay was performed via the newly proposed BL inequalities. A numerical example illustrated the efficiency of the results. One of the directions for the future research is extension of the obtained results to the KdVB equation with time-delay [20].

\section{REFERENCES}

[1] Z. Hidayat, R. Babuska, B. De Schutter, and A. Nunez, "Observers for linear distributed-parameter systems: A survey," in 2011 IEEE International Symposium on Robotic and Sensors Environments (ROSE), pp. 166-171, IEEE, 2011.

[2] E. Fridman and A. Blighovsky, "Robust sampled-data control of a class of semilinear parabolic systems," Automatica, vol. 48, no. 5, pp. 826-836, 2012.

[3] N. Bar Am and E. Fridman, "Network-based $H_{\infty}$ filtering of parabolic systems," Automatica, vol. 50, no. 12, pp. 3139-3146, 2014.

[4] R. Katz, E. Fridman, and A. Selivanov, "Boundary delayed observercontroller design for reaction-diffusion systems," IEEE Transactions on Automatic Control, 2020, DOI: 10.1109/TAC.2020.2973803.

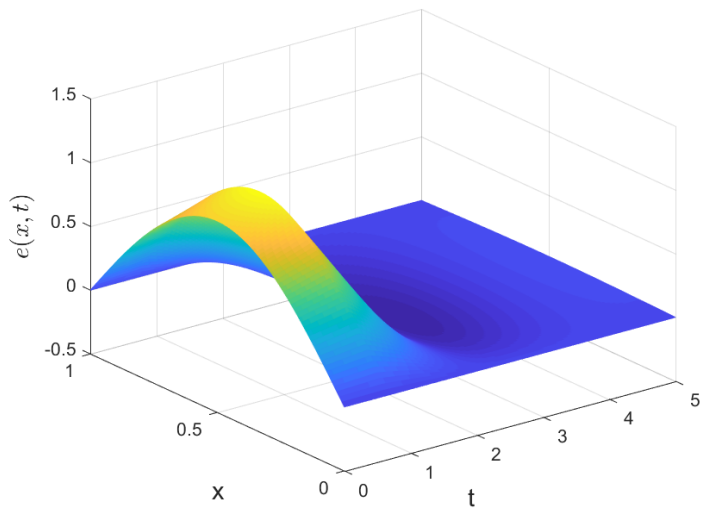

Fig. 2. The error $e(x, t)$.

[5] T. Ahmed-Ali, E. Fridman, F. Giri, M. Kahelras, F. LamnabhiLagarrigue, and L. Burlion, "Observer design for a class of parabolic systems with large delays and sampled measurements," IEEE Transactions on Automatic Control, 2019, DOI: 10.1109/TAC.2019.2941434.

[6] K. Gu, "An integral inequality in the stability problem of time-delay systems," in Proceedings of the 39th IEEE Conference on Decision and Control, vol. 3, pp. 2805-2810, IEEE, 2000.

[7] K. Gu, J. Chen, and V. L. Kharitonov, Stability of time delay systems. Boston: Birkhäuser, 2003.

[8] A. Seuret and F. Gouaisbaut, "Wirtinger-based integral inequality: Application to time-delay systems," Automatica, vol. 49, no. 9, pp. 28602866, 2013.

[9] P. Park, W. I. Lee, and S. Y. Lee, "Auxiliary function-based integral inequalities for quadratic functions and their applications to time-delay systems," Journal of the Franklin Institute, vol. 352, no. 4, pp. 13781396, 2015.

[10] H.-B. Zeng, Y. He, M. Wu, and J. She, "Free-matrix-based integral inequality for stability analysis of systems with time-varying delay," IEEE Transactions on Automatic Control, vol. 60, no. 10, pp. 2768$2772,2015$.

[11] A. Seuret and F. Gouaisbaut, "Hierarchy of LMI conditions for the stability analysis of time-delay systems," Systems \& Control Letters, vol. 81, pp. 1-7, 2015.

[12] L. Baudouin, A. Seuret, and F. Gouaisbaut, "Stability analysis of a system coupled to a heat equation," Automatica, vol. 99, pp. 195C202, 2019.

[13] T. Wang, "Stability in abstract functional differential equations. part II. Applications," Journal of Mathematical Analysis and Applications, vol. 186, pp. 835-861, 1994.

[14] M. Tucsnak and G. Weiss, Observation and control for operator semigroups. Birkhäuser, 2009.

[15] E. Fridman, Introduction to time-delay systems: analysis and control. Basel: Birkhäuser, 2014.

[16] R. E. Bellman and K. L. Cooke, "Differential-difference equations," 1963.

[17] D. Henry, Geometric theory of semilinear parabolic equations. New York: Springer-Verlag, 1981.

[18] W. Gautschi, "Orthogonal polynomials: Computation and approximation. 2004," Numerical Mathematics and Scientific Computations, 2004.

[19] A. Seuret, F. Gouaisbaut, and Y. Ariba, "Complete quadratic lyapunov functionals for distributed delay systems," Automatica, vol. 62, pp. 168-176, 2015.

[20] W. Kang and E. Fridman, "Distributed stabilization of Korteweg-de Vries-Burgers equation in the presence of input delay," Automatica, vol. 100, pp. 260-273, 2019. 